\author{
R. Fanti \\ Istituto di Fisica and Laboratorio di Radioastronomia, Bologna \\ G.C. Perola \\ Istituto di Scienze Fisiche dell'Università, Milano
}

\title{
1. DEFINITIONS
}

The monochromatic luminosity function of radio sources (RLF) is the number of sources per unit volume as a function of the luminosity $P$ at a frequency $v$ and of the cosmic epoch $(z)$. Symbol: $n(P(\nu), z)$. It is often given per interval of $\log P$, or $M_{Y}$, the absolute radio magnitude. This function is determined only for sources associated with optical objects (galaxies and QSO's). It can be given for all kinds of associations, or for sources associated with a specific type of object. In this case the normalized, or fractional, RLF is sometimes used, $F_{i}(P, z)=n_{i}(P, z) / \rho_{i}(z)$, where $\rho_{i}$ is the spare density of type $i$ objects. The word "bivariate" is used for the RLF defined per interval of the optical luminosity (or mag-nitude M). A RLF can be determined using either a radio-optically complete sample of identified sources, or the radio observation of an opti-cally selected sample. The merits of methods used to estimate a RLF from a complete sample are discussed by Felten (1976). Translation of a RLF from one frequency to another must be done with care if, at the two frequencies, different radio components (like the extended and the compact) would be preferentially sampled. We shall review the estimates of local $(z=0)$ RLF's using $\mathrm{H}_{\mathrm{O}}=100 \mathrm{Kms}^{-1} \mathrm{Mpc}^{-1}$ and the unit WHz $\mathrm{W}^{-1}$ for $\mathrm{P}$.

\section{OVERALL RLF}

Wall, Pearson and Longair (this volume) use a sample of 87 3CR sources with $\mathrm{S}(408 \mathrm{MHz}) \geq 10 \mathrm{Jy}$, of which 74 are identified with galaxies and QSO's having a measured redshift. Thus, they obtain a luminosity distribution that extends down to $\log \mathrm{P}(408)=21$ with the help of Cameron (1971) and Caswell and Wills (1967) (see Sections 3 and 4). The $n(P, z)$ is then estimated by fitting both this distribution and a distribution of source counts down to $\mathrm{S}(408)=12 \mathrm{mJy}$. The best fit of $\mathrm{n}(\mathrm{P}, 0)$ in $\mathrm{a}$ Friedmann universe with $\mathrm{q}_{0}=1 / 2$ is shown in Fig. 1 . This procedure must be followed for sources with $\log P>26$, whose local densities are too low for a direct determination. Below this power a local $\mathrm{n}(\mathrm{P})$ for galaxies can be constructed using sources within $z=0.1$, where evolutionary effects can be neglected and the choice of the universe geometry 
is not critical. We shall consider separately the contribution of the elliptical and So galaxies, $(E+S O)$, and of the spiral and irregular galaxies, $(S+1)$.

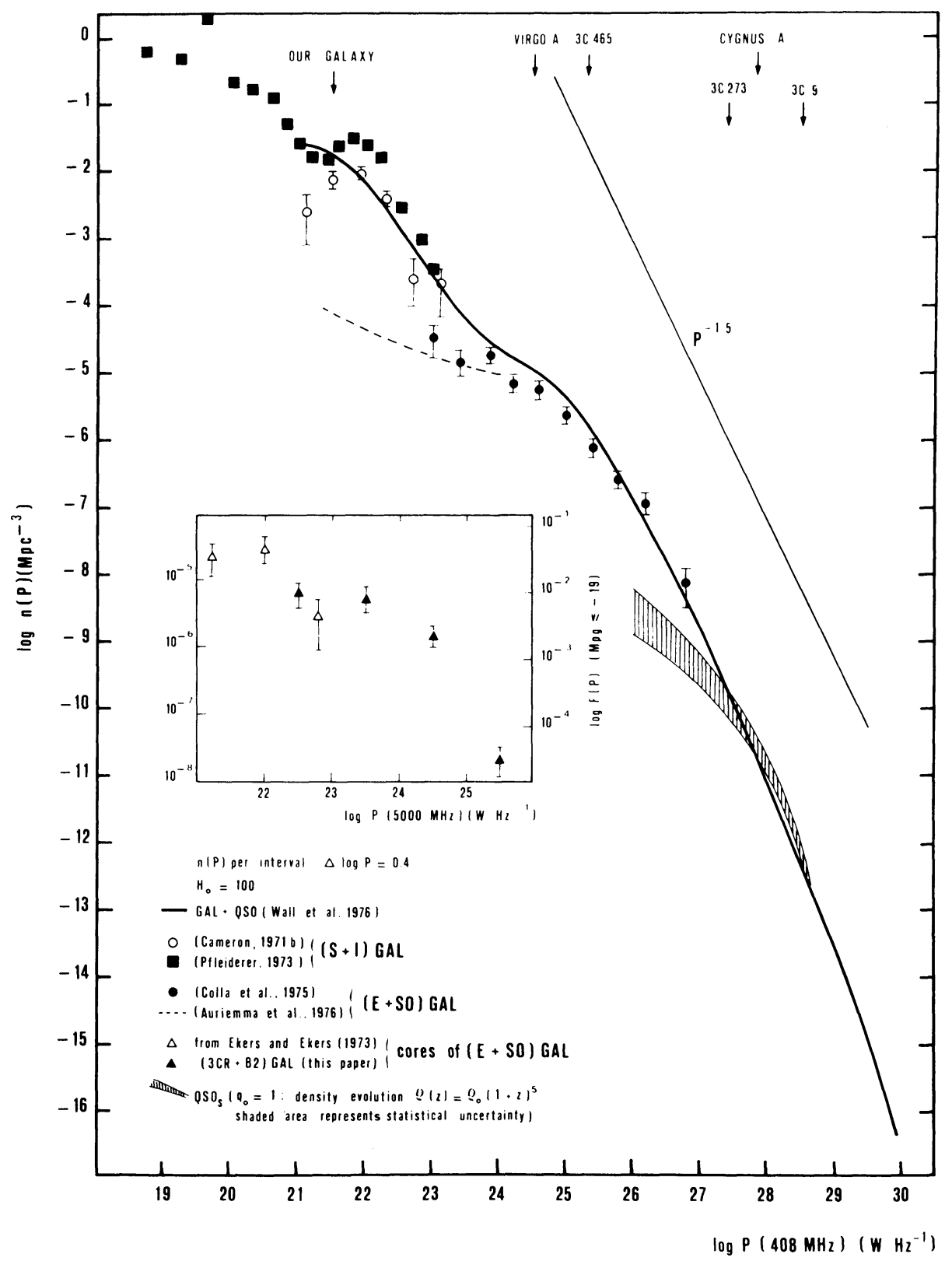

Fig. 1. The overall Radio Luminosity Function. 


\section{3. $(E+S O)$ GALAXIES}

Colla et a1. (1975) use a sample of 53 B2 sources ( $(408)>0.20 ; 0.25 \mathrm{Jy})$ and 53 3CR sources $(\mathrm{S}(408)>5 \mathrm{Jy})$ identified with $\mathrm{E}$ and so galaxies brighter than $\mathrm{m}_{\mathrm{p}}=15.7$ and $\mathrm{m}_{\mathrm{v}}=17$, respectively (the redshift available for all galaxies). Their RLF is given in Fig. 1 along with an extension down to $\log P=21.5$ by Auriemma et al. (1976; see Section 5) (error bars here and later are equal to $\mathrm{N}^{-1 / 2}$ percent). No attempt is made to separate the $\mathrm{E}$ from the SO. Note the rather sharp change of slope at about $\log \mathrm{P}=$ $=25$. Earlier estimates of $n(P)$ for $\log P>23$, where the spiral contribution is negligible (Caswell and Wills, 1967; Merkelijn, 1971), are not as reliable because the distances were mostly estimated indirectly using a fixed absolute magnitude for radio galaxies (see Section 5). However, the flattening below $\log P=25$ seen by Caswell and Wills but not by Merkelijn is confirmed.

\section{4. $(S+I)$ GALAXIES}

Cameron (1971a,b) observed an optically complete sample of bright galaxies $(\mathrm{m}<11.0 ; 12.5)$ at $408 \mathrm{MHz}$ and detected a total of 85 galaxies with known distance. 72 of these are $(S+I)$ and have $\log \mathrm{P}<23.5$; the corresponding RLF is shown in Fig. 1. The contribution of this type of galaxy peaks at $\log \mathrm{P}=22$ and drops to a density comparable to that of the $(E+\mathrm{SO})$ type at about $\log \mathrm{P}=23.5$. The results of a similar work by Pfleiderer (1973) at $1.4 \mathrm{GHz}$ on a larger sample of galaxies (twice as many detections with known distance) are also given in Fig. 1 (the RLF has been shifted to $408 \mathrm{MHz}$ using a mean spectral index $=0.75)$. The discrepancy with Cameron's result is probably due to a rather uncertain correction for incompleteness. However, the peak at $\log \mathrm{P}=22$ is confirmed. The extension to lower luminosities shows a minimum at about $\log P=21$, follow-ed by a new rise. Pfleiderer's data indicate that this structure may be due to a different form of the RLF of the S and I galaxies, the irregulars becoming dominant at $\log \mathrm{P}<21$. This part of the curve, however, is very uncertain due to the limited number of detections available.

\section{CORRELATIONS BETWEEN OPTICAL AND RADIO LUMINOSITY IN GALAXIES}

To investigate this property, the $(E+S O)$ bivariate RLF has been studied by Ekers and Ekers (1973) at $5 \mathrm{GHz}$ (see Section 7) and by Colla et al. (1975) at $408 \mathrm{MHz}$. Auriemma et al. (1976) extend the latter work by combining the $B 2$ and $3 C R$ samples with a sample of 17 radio galaxies in rich clusters (Jaffe and Perola, 1976) and a sample of 23 non-cluster radio galaxies (Ekers et al. 1976) to obtain a bivariate $F(P)$ at $1.4 \mathrm{GHz}$ for $M_{p} \leq-18$ per interval of one magnitude. Fig. $2 a$ shows the result of fitting power laws to the data. Note the break in the slope for each interval of $\mathrm{M}_{\mathrm{p}}$ at about the same power $\log \mathrm{P}_{1.4}^{*}=24.5$; at $\operatorname{larger} \mathrm{P}$ the slope is equal to $1.3 \pm .1$ independent of $M_{p}{ }^{1}{ }_{4}^{4}$ to $\log \mathrm{P}=26$; at smaller $\mathrm{P}$ the slope decreases with $\mathrm{M}_{\mathrm{P}}$. The latter property reflects the approach to the saturation (100\%) of the integral value of $F(P)$. Above $\mathrm{P}^{*}$ the 


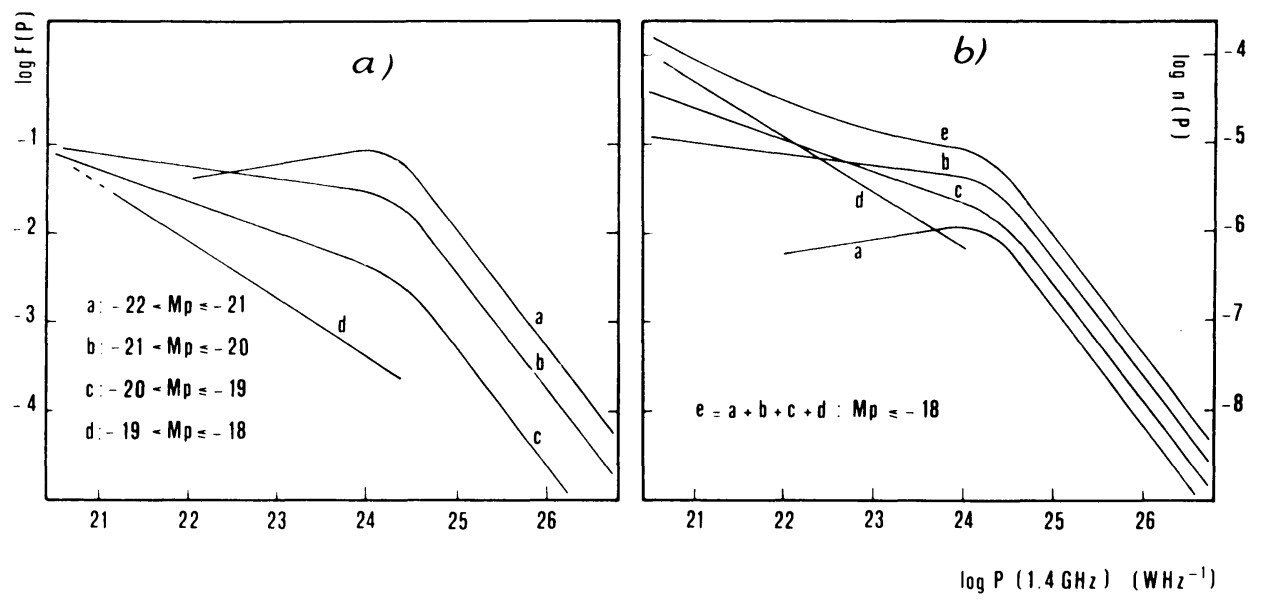

Fig. 2. a) bivariate fractional RLF and b) bivariate RLF.

$F(P)$ depends on the optical luminosity $L$ as $\mathrm{L}^{-1 \cdot 5}$. From the bivariate $\mathrm{n}(\mathrm{P})$ (Fig. 2b) a most probable value of $\mathrm{M}_{\mathrm{p}}=-20.3$ for radio galaxies is derived for $P>P^{*}$, independent of $P$, while this value increases as $P$ decreases for $\mathrm{P}<\mathrm{P}^{*}$. The first result corresponds to that obtained by Sandage (1972) for a sample of $3 \mathrm{CR}$ sources, $\left\langle\mathrm{M}_{\mathrm{v}}\right\rangle=-21.48$, and shows that the use of a constant $<M>$ to construct a distance modulus for radio galaxies is justified locally only for $24.5<\log \mathrm{P}_{1} 4^{<}<26$. Beyond $\log \mathrm{P}=26$ a purely local determination of $\left\langle\mathrm{M}>\right.$ is not $\mathrm{t}^{4}$ possible for statistical reasons, while evolutionary effects might change its value at $z>0.1$.

For the $(S+I)$ galaxies, using the data from Cameron (1971a), one finds that the bivariate peaks at about $\log \mathrm{P}_{408}=22$ for $-21<\mathrm{M}_{\mathrm{p}} \leq-20$ and $-20<M_{p} \leq-19$, and $\log \mathrm{P}_{408}=21.4$ for $-19<\mathrm{M}_{\mathrm{P}} \leq-18$, showing that the average radio luminosity is larger for the brighter galaxies.

\section{GALAXIES INSIDE AND OUTSIDE CLUSTERS}

The results of the observations of the $(E+S O)$ in 5 rich clusters by Jaffe and Perola (1976) at $1.4 \mathrm{GHz}$ are compared by Auriemma et al. (1976) with those obtained also at $1.4 \mathrm{GHz}$ by Ekers (1976) on a sample of $(E+S O)$ galaxies outside rich clusters (but still mostly in aggregates of various sizes). In Fig. 3 the two $F(P)$ for $M_{p} \leq-19$ are shown. To take care of the effects of the dependence on $M$ of the bivariate $F$, in constructing the $F(P)$ from the second sample its optical luminosity distribution has been normalized to that of the cluster sample. From the comparison there seems to be no systematic difference in the two function below $\log \mathrm{P}_{1.4}=24$. Above this power, Riley (1976), from a critical 


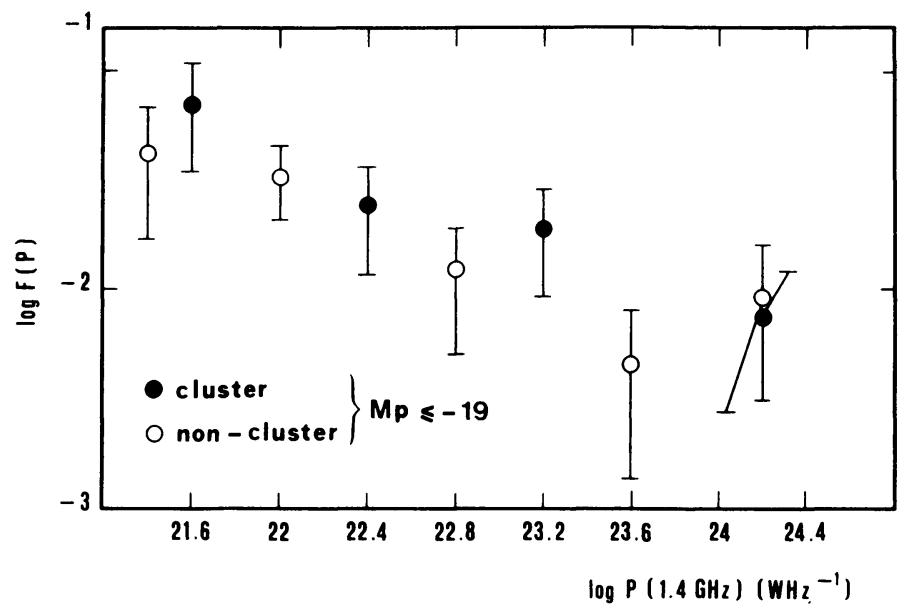

Fig. 3. RLF for cluster and non-cluster radio galaxies.

analysis of the identification of sources in various surveys with Abell clusters, concludes that a) the RLF of those sources is similar in form to the general one from the 3CR, and that between $1 / 10$ and $1 / 4$ (this gives a measure of the uncertainties) of all radio sources up to $\log \mathrm{P}_{1.4}=$ $=26.5$ lie in Abell clusters; b) galaxies brighter than $\mathrm{M}_{\mathrm{p}}=-20$ have the same probability of being a radio source inside as well as outside Abell clusters; and c) no correlation is evident between the richness of a cluster and its probability of containing a source. On the other hand Owen (1975), on the basis of observations of over 500 Abell clusters with the 300 foot NRAO telescope at $1.4 \mathrm{GHz}$, finds a form of the RLF that peaks at $\log \mathrm{P}_{1,4} \simeq 24.5$. He tentatively explains this feature as being due to the summation in its beam (10') of the radio emission of more than one galaxy per cluster (see also Jaffe in this volume). McHardy (1974), using $3 \mathrm{CR}$ and $4 \mathrm{C}$ sources in clusters, finds that powerful radio sources tend to be more common in Bautz-Morgan class I clusters, a property which does not seem to be explicable only with the difference in the bright end of the optical luminosity function between class I and the other BM classes. Owen (1975) finds an analogous trend (higher detection probability of clusters dominated by a giant central galaxy) using the RoodSastry classification.

For the (S + I) Jaffe and Perola (1976) (see also Jaffe et al. 1976) find evidence that the spirals in Coma (a spiral poor cluster) are more powerful relative both to the nearby spirals and to those in a spiral rich cluster like Hercules. More clusters need to be studied to see if this is a general property differentiating spiral poor from spiral rich clusters. 


\section{STATISTICS ON COMPACT SOURCES IN ELLIPTICAL GALAXIES}

High resolution, high frequency observations show the existence of small diameter (< 1-2 arcsec) radio sources (cores) in the central region of elliptical galaxies. These cores are generally found either by high frequency observations of nearby elliptical galaxies (e.g. Heeschen, 1970; Ekers and Ekers, 1973) or by high frequency, high resolution observations of previously known radio galaxies (e.g. Pooley and Henbest, 1974; Riley and Pooley, 1975; Colla et al. 1975).

The physical sizes of these cores, as derived from VLBI observations, range from $\sim 1$ parsec (e.g. Cyg A, Kellermann et al. 1975) to about $1 \mathrm{Kpc}$ (e.g. 3C 236, Fomalont and Miley, 1975). However, the actual size distribution is not known due to the incompleteness of the sample studied with the VLBI technique. Typically core sources of "Cyg $A$ " type have a flat spectra, while core sources of "3C 236 type" have normal radio spectra. These cores are often considered as a manifestation of the explosive phenomena occurring in the nuclei of elliptical galaxies, which ultimately supply energy to the extended component of the radio source. A statistical study of the properties of cores could shed light on the problem of how extended radio sources are formed and then powered during their evolution.

With the data presently available in the literature we obtain: a) the distribution of the ratio $\underline{r}=P_{\text {ext. }} / \mathrm{P}_{\text {core }}$ as a function of the total radio luminosity $\mathrm{P}_{\text {tot }}$; b) the $\mathrm{RLF} \mathrm{n}(\mathrm{P})$ of the cores in elliptical galaxies; and $c$ ) the frequency of cores as a function of the optical luminosity of the parent galaxy.

The present analysis is based on: i) a sample of 53 elliptical radio galaxies from the B2 catalogue as studied by Colla et al. (complete sample) ii) a sample of 34 radio galaxies brighter than $\mathrm{m}_{\mathrm{v}}=17.0$, from the $3 \mathrm{CR}$ catalogue (almost a complete sample) and an incomplete subset of 25 optically weaker 3CR radio galaxies. All these radio galaxies have been observed at $5 \mathrm{GHz}$ at Westerbork and Cambridge (see references above). Several core sources have been detected $(31,20$ and 9 , respectively) among them corresponding to percentages ranging from 35 to $60 \%$. Since many cores are near the detection limits, we expect that there may be more which have not been detected, especially among more distant galaxies. From the available maps it is possible to estimate upper limits on the intensity of undetected core sources. On the other hand, for radio galaxies which appear "core-like" (9 in the B2 sample and 1 in the 3CR sample), we have estimated an upper limit to the intensity of an extended component.

a) Through proper use of the upper limits on $\underline{r}$, we estimate the "true" percentage of cores in radio galaxies, as a function of $\mathrm{P}_{\text {tot }}(408 \mathrm{MHz})$ and of the ratio $\underline{r}$ (at $5 \mathrm{GHz}$ ). The result is given in Table 1. Each box contains the ratio between cores which could have been detected if they had equal or smaller $\underline{r}$ values. Table 1 shows: $i)$ for each interval of $P_{\text {tot }}$ the fraction of radio galaxies with a core is $>70 \%$; ii) the 
core luminosity does not seem to increase with the total luminosity. However, a correlation of the type $\mathrm{P}$ core $\propto \mathrm{P}$ tot is equally possible as a null correlation. This result, if subsequently confirmed, is rather important in connection with the problem of an energy supply to the extended radio components. It would seem that the core luminosity is not a good indicator of the energy supply required to maintain the current luminosity of the extended component.

b) We obtain a RLF at $5 \mathrm{GHz}$ of cores of elliptical galaxies from Table 1 and from the RLF of radio galaxies. It is displayed in Fig. 1, along with that derived from the survey at $5 \mathrm{GHz}$ of nearby elliptical galaxies by Ekers and Ekers. Because the selection was made at a much lower frequency, where the extended components dominate, our determination may be biased against core-like flat spectrum sources. However, agreement with the results of Ekers and Ekers shows that this selection effect is not significantly large and that most of the cores are therefore associated with extended components. In the range $21<\log \mathrm{P}_{5000}<24$, about 50\% of the cores are associated with extended components of a similar or stronger radio luminosity (as noted also by Ekers and Ekers). The RLF of the cores seems to have a break around $\log \mathrm{P}_{5000} \simeq 24.5$, as does the general RLF. The slope beyond the break is not well determined, but it may be even steeper than that of the general RLF since there are no known core radio galaxies much stronger than $\log \mathrm{P}_{5000} \simeq 25$. A determination of the cores RLF at these radio luminosities should be derived in the future from optical identifications of radio sources in the $5 \mathrm{GHz} \mathrm{NRAO}$ radio survey.

c) Colla et al. (1975), using $5 \mathrm{GHz}$ Westerbork observations of the B2 sample, gave evidence that the frequency of cores in elliptical galaxies depends on the optical luminosity of the parent galaxy in a way similar to the overall radio emission. Adding the galaxies of the 3CR samples, for which accurate magnitudes are available, confirms this result. Specifically, the fraction of ellipticals in the ranges $\left(-22<\mathrm{M}_{\mathrm{p}}<-21\right)$, $\left(-21<\mathrm{M}_{\mathrm{p}}<-20\right),\left(-20<\mathrm{M}_{\mathrm{p}}<-19\right)$, with cores having 1 og $\mathrm{P}_{5000}>22.8$, is $(19 \pm 10) \%,(6 \pm 2) \%$ and $(1 \pm .5) \%$, respectively.

Table 1

Percentages of Cores of Different $r$ as Function of $P_{\text {tot }}$

\begin{tabular}{rrrrc}
\hline $\log r$ & $2.5 \pm .5$ & $1.5 \pm .5$ & $.5 \pm .5$ & $<0.0$ \\
\hline $10 g P_{\text {tot }}$ & & & & \\
$>26$. & $29 \pm 14 \%$ & $34 \pm 12 \%$ & $4 \pm 4 \%$ & \\
$25.5 \pm .5$ & $5 \pm 5 \%$ & $30 \pm 11 \%$ & $20 \pm 9 \%$ & $23 \pm 9 \%$ \\
$24.5 \pm .5$ & $13 \pm 9 \%$ & $20 \pm 11 \%$ & $25 \pm 11 \%$ & $24 \pm 11 \%$ \\
$23.5 \pm .5$ & & $8 \pm 8 \%$ & $34 \pm 14 \%$ & $24 \pm 11 \%$ \\
\hline
\end{tabular}




\section{QUASARS}

It is not possible to determine a local RLF for quasars, since their local density $(z<0.1)$ is too low for a direct determination. Al1 of our information comes from objects at larger distances $(z>0.2)$, where evolutionary effects are dominant. Therefore, a determination of the RLF of quasars at the present epoch is strictly connected to the determination of their evolution law. Evidence concerning the evolution of quasars has been reviewed by Schmidt (1972a) who showed that it can be accounted for by a pure density evolution described either by an exponential law $\rho(z)=\rho_{0} 10^{5 \tau}$ ( $\tau$ being the look-back time in units of the age of the universe) or by a power law $\rho(z)=\rho_{0}(1+z)^{n}$, with $n=6 \pm 1$.

We assume, in that which follows, the second expression with $\mathrm{n}=5$. From the complete samples of radio quasars (QSS's) available in the 1iterature, 3CR (see Smith et al. 1976) and 4C (Lynds and Wills, 1972), a RLF extrapolated to $z=0$, by means of the assumed evolution, is derived This is shown in Fig. 1. A comparison with the overall RLF of radio sources shows that, for $\log \mathrm{P}_{403}>27.5$, quasars contribute to most of the space density of radio sources, while at $\log \mathrm{P}_{408}<27.0$, the contribution of the radio galaxies is dominant (see also Schmidt, 1972b). Chang of $\mathrm{n}$ by \pm 1 would change the local space density of quasars by about a factor 3 at the bright end but would not change the lower end significant 1 The total space density of quasars down to $\log \mathrm{P}_{408} \simeq 26.0$ is $(10 \pm 2.5)$. - $10^{-9} \mathrm{Mpc}^{-3}$. This value may be compared with the density of all quasars obtained from optically selected samples regardless of their radio emissio (QSO's) (Braccesi et al. 1970; Schmidt, 1970). The optical luminosity function of quasars, determined from these optical samples adopting the same evolution law as for the radio quasars, is a power law with slope $1.5 \pm .15$. Above an absolute optical luminosity, at $2500 \AA, F_{2500}=10^{22}$. the integrated space density, at zero redshift, is $(1.3 \pm .4) \cdot 10^{-7} \mathrm{Mpc}^{-3}$ and above $\log \mathrm{F}_{2500}=21$ it might be up to $\simeq 10^{-5} \mathrm{Mpc}^{-3}$. It has been shown (Fanti et a1. 1973) that strong radio emission is a property of only those quasars with $\log \mathrm{F}_{2500}>22.4$, while optically weaker quasars are very seldom strong radio emitters. Thus, it appears that only about $8 \%$ of al1 quasars with $\log \mathrm{F}_{2500}>22.4$ are radio sources with radio luminosity larger than $10^{26}$.

Several attempts have been made to extend the determination of the RLF at lower radio luminosities. Most of these attempts are done through the use of the so-called $\Psi(R)$ function. This represents the fraction of all quasars, per unit volume, having ratio $\mathrm{R}$ between the monochromatic radio and optical luminosities at $500 \mathrm{MHz}$ and at $2500 \AA$, respectively (see Schmidt, 1970). The introduction of this function came from Schmidt' finding (1970) that the redshift distributions at the 18 th magnitude for QSS's and QSO's are very similar, which seemed to imply that the radio and optical luminosities are correlated. $\Psi(R)$ may be determined on the basis of complete samples of QSS's, by knowing the number-magnitude, $N(<\mathrm{m})$ relationship for QSO's (see Schmidt, 1970; Fanti et a1. 1973) and by the formula

$$
\Psi(R) \cdot \Delta R=\frac{N(<m, r)}{N(<m)}
$$


where $\mathrm{N}(<\mathrm{m}, \mathrm{r})$ is the number of CSS's in the considered sample which are brighter than $\mathrm{m}$ and possess a radio to optical flux ratio equal to $\mathrm{R} \pm$ $\pm \Delta R / 2$. Alternatively, it $c$ an be found by searching radio emission from optically selected quasars. $\Psi(R)$ is reasonably well determined for

$\log \mathrm{R}>3.5$ from the $3 \mathrm{CR}$ and $4 \mathrm{C}$ samples. Attempts to determine $\Psi(\mathrm{R})$ below that value have been made in the past by Fanti et al. (1973, 1975) and Katgert et al. (1973) on the basis of: i) a sample of quasars from the B2 catalogue; $i$ i) a search of radio emission down to $\simeq 5-10 \mathrm{mJy}$, from the sample of QSO's studied by Braccesi et a1. (1970).

From the first sample it appeared that $\simeq 30-100 \%$ of quasars (with $\log F_{2500}>22.4$ ) have radio luminosities with $\log \mathrm{R}>2.0$. From the second sample it was determined that at most $20 \%$ of all quasars have $\log R>2.0$. Since the two determinations of $\Psi(R)$ were thought to be significantly different, and were also obtained from two samples at different depths in space, the discrepancy was assumed as evidence of an evolutionary effect in quasars with $2.0<\log \mathrm{R}<3.0$ (Fanti et al. 1973, 1975). Recently other attempts have been made to determine $\Psi(R)$, at $\log \mathrm{R}<3.0$, using: i) a sample of QSS's from the Westerbork survey (de Ruiter, Willis and Arp, 1976); ii) a search for radio emission from a new sample of 51 QSO's studied spectroscopically by Schmidt (1974) plus 8 bright QSO's from the Tonantzintla catalogue (Iriarte and Chavira, 1957). This search led to 8 radio detections above $10 \mathrm{mJy}$ (Fanti et al. 1976). This figure is in excellent agreement with the previous result by Katgert et al. concerning the QSO's studied by Braccesi et al. The various determinations of $\Psi(\mathrm{R})$ are shown in Fig. 4 (again only for those quasars with $\left.\log \mathrm{F}_{2500}>22.4\right)$. The new radio data on QSO's have been combined with the previous measures by Katgert et al. There is still a systematic difference (at $\sim 2 \sigma$ level) between the $\Psi(R)$ determinations from the optically selected and the radio selected samples in the range $2.0<\log \mathrm{R}<$ $<3.0$. However, the previously suggested explanation of an evolutionary effect has now been ruled out, since most of the new radio observed QSO's span the same range of apparent optical luminosity and, therefore, of redshift as the radio samples. The difference may be just a large statistical fluctuation or it may be due to an incorrect $\mathrm{N}(<\mathrm{m})$ relationship at the very bright end $(16.0<\mathrm{m}<17.5)$, which is used to compute the $\psi(\mathrm{R})$, and, therefore, to an evolutionary behaviour at small redshifts $(z<0.5)$ different from the assumed power law.

If we assume the determination from the QSO samples in the range $2.0<\log \mathrm{R}<3.0$ as the best estimate of $\Psi(\mathrm{R})$, we see that at $\log \mathrm{R}>$ $>1.5$ only about $17 \%$ of all quasars are radio emitters. Presumably, the bulk of them have $\log \mathrm{R}$ values much lower than 1.0. $\Psi(\mathrm{R})$ might also have a maximum near $\log R=2.5$ and then decrease at low $R^{\prime} s$. This shows that the phenomenon of strong radio emission is not more frequent among the quasars than it is among the elliptical galaxies. We are led to think either that the radio emission is a transient phenomenon in the life of quasars or that only in a small number of cases the physical conditions favour a strong emission at radio wavelengths. 


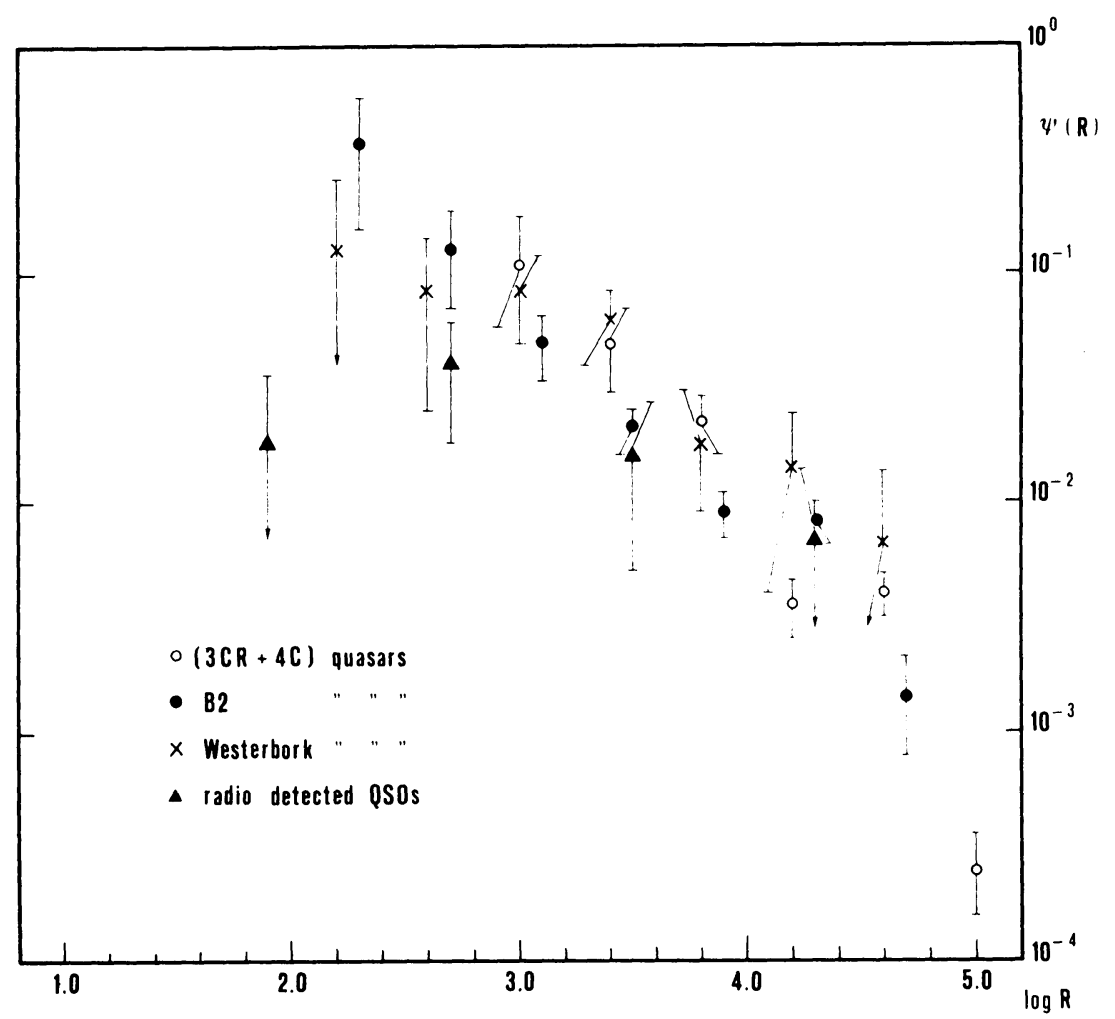

Fig. 4. The $\Psi($ R) function.

\section{REFERENCES}

Auriemma, C., Perola, G.C., Ekers, R.D., Fanti, R., Lari, C., Jaffe, W.J. and Ulrich, M.-H., 1976, in preparation.

Braccesi, A., Formiggini, L. and Gandolfi, E., 1970, Astron. Astrophys. $24,247$.

Cameron, M.J., 1971a, Mon. Not. Roy. Astron. Soc. 152, 403.

Cameron, M.J., 1971b, Mon. Not. Roy. Astron. Soc. $\overline{152}, 429$.

Caswe11, J.L. and Wil1s, D., 1967, Mon. Not. Roy. Astron. Soc. 135, 231.

Colla, G., Fanti, C., Fanti, R., Gioia, I., Lari, C., Lequeux, J., Lucas, R. and U1rich, M.-H., 1975, Astron. Astrophys. 38, 209.

Ekers, R.D. and Ekers, J.A., 1973, Astron. Astrophys. 24, 247.

Ekers, R.D., Ekers, J.A., Rogstad, D.R. and Smeding, A., 1976, in preparation.

Fanti, C., Fanti, R., Ficarra, A., Formiggini, L., Giovannini, G., Lari, C. and Padriel1i, L., 1975, Astron. Astrophys. 42, 365.

Fanti, C., Fanti, R., Lari, C., Laan, H. van der, Padriel1i, L. and de Ruiter, H.R., 1976, in preparation.

Fanti, R., Formiggini, L., Lari, C., Padrie11i, L., Katgert-Merkelijn, J.K. and Katgert, P., 1973, Astron. Astrophys. 23, 161. 
Felten, J.E., 1976, submitted to Astrophys. J.

Fomalont, E.B. and Miley, G.K., 1975, Nature 257, 99.

Heeschen, D.S., 1970, Astrophys. Letters 6, 49 .

Iriarte, B. and Chavira, E., 1957, Bu11. Obs. Ton. No. 16, 3.

Jaffe, W.J. and Perola, G.C., 1976, Astron. Astrophys. 46, 275.

Jaffe, W.J., Perola, G.C. and Valentijn, E.A., 1976, Astron. Astrophys.

$49,179$.

Katgert, P., Katgert-Merkelijn, J.K., Le Poole, R.S. and Laan, H. van der, 1973, Astron. Astrophys. 23, 171 .

Kellermann, K.I., Clark, B.G., Nie11, A.E. and Shaffer, D.B., 1975, Astrophys. J. Letters 197, L113.

Lynds, R. and Wills, D., 1972, Astrophys. J. 172, 531.

McHardy, I.M., 1974, Mon. Not. Roy. Astron. Soc. 169, 527.

Merkelijn, J.K., 1971, Astron. Astrophys. 15, 11.

Owen, F.N., 1975, Astrophys. J. 195, 593.

Pfleiderer, J.L., 1973, Mitt. Astron. G. 32, 108.

Pooley, G.G. and Henbert, S.N., 1974, Mon. Not. Roy. Astron. Soc. 169, 477 Riley, J.M., 1975, thesis.

Riley, J.M. and Pooley, G.G., 1975, Mem. Roy. Astron. Soc. 80, 105.

Ruiter, H.R. de, Willis, A.G. and Arp, H.C., 1976, submitted to Astron.

Astrophys. Suppl.

Sandage, A., 1972, Astrophys. J. 178, 25.

Schmidt, M., 1970, Astrophys. J. $\overline{162}, 371$.

Schmidt, M., 1972a, Astrophys. J. $\overline{176}, 273$.

Schmidt, M., 1972b, Astrophys. J. $\overline{176}, 303$.

Schmidt, M., 1974, Astrophys. J. 1하, 509.

Smith, H.E., Spinrad, H. and Smith, E.0., 1976, submitted to Publ. Astr.

Soc. Pacific.

\section{DISCUSSION}

H.E. Smith: If I understand correctly, your luminosity function requires that $\left\langle M_{p}>\right.$ for powerful radio galaxies increase by 0.4 per decade of radio power. However, if you look at $3 \mathrm{CR}$ galaxies with $\mathrm{m}<19^{\prime}$ where there is some measure of completeness, then there is a difference of les, than 0.3 mag over about 4 decades of radio power.

Perola: I have shown that $\left\langle M_{p}\right\rangle$ depends on the power of the sources onl if $\mathrm{P}$ is less than $10^{24} \mathrm{WHz}^{-1}$ and that at larger powers $\left\langle\mathrm{M}_{\mathrm{p}}\right\rangle$ is independec of $P$. This result is in full agreement with what you say about $3 \mathrm{CR}$ galaxies, whose powers, at $z$ larger than 0.03 , are larger than $10^{24.5}$. In fact the result you mention also indicates that $\left\langle\mathrm{M}_{\mathrm{p}}\right\rangle$ in the large power range stays about constant beyond the limit $z=0.1$ imposed in the determination of the bivariate $n(P)$ in our Fig. $2 b$.

Conway: Data by Edwards at Jodrell Bank gives the power emitted by central components vs. the power emitted by the whole source for 26 bright $3 \mathrm{C}$ sources, and suggests that these are proportional to each other for radio galaxies. The ratio is higher for quasars.

R. Fanti: If one plots core luminosity against luminosity of the whole 
source, one actually finds a proportionality between the two luminosities. But this is largely due to a selection effect. More powerful radio galaxies are generally far away and therefore, owing to the limited sensitivity of radio telescopes, one detects only stronger cores.

Rowan-Robinson: I have tried to tie together what we know about activity in galaxies - radiogalaxies, N-galaxies, seyferts and quasars - into a single picture. I assume that the probability of a galaxy being active depends on optical luminosity, declining from 100 for the most luminous to 28 for the least luminous. I then construct probability functions for the luminosity of radio cores, optical cores, extended radio sources (for active ellipticals) and disc emission (for all spirals). I assume a range of dust optical depths in front of the optical cores to explain the infrared emission from active galaxies.

QSOs are then simply active galaxies whose optical cores outshine the parent galaxy, while in seyferts the optical core has to contribute at least 18 of the total light. The distribution functions for the various observable parameters fit most of what we know about active galaxies (the bulk of the data reviewed by Fanti \& Perola, for example). A surprising prediction is that most optically selected QSOs are in spirals, explaining their low radio to optical ratio. Although the scheme has yet to be evolved to earlier epochs, a prediction could be that compact, flat spectrum radio sources do not show much evolution. 